\title{
Chapter 9 \\ Conclusions
}

This thesis presented fiducial and differential cross section measurements of the Higgs boson at $\sqrt{s}=8 \mathrm{TeV}$ with the ATLAS detector, and fiducial and total cross section measurements of $W Z$ diboson production at $\sqrt{s}=13 \mathrm{TeV}$. The electron likelihood method of identification, first introduced in Run 1 and adapted for use in Run 2, is described in detail. Electron efficiency measurements of identification criteria are described as well, featuring methods developed in Run 1 and employed during Run 2 data taking.

The Higgs fiducial cross section and decay to four leptons $(e$ or $\mu)$ at $8 \mathrm{TeV}$ was measured to be $\sigma_{\text {fid }}=2.11_{-0.47}^{+0.53}$ (stat) \pm 0.08 (syst) fb, and can be compared to the theoretical prediction [1] of a Higgs with mass $125.4 \mathrm{GeV}$ of $1.30 \pm 0.13 \mathrm{fb}$. Unfolded differential distributions of kinematic observables show no statistically significant deviation from the SM predictions according to a number of state-of-theart generators.

The $W Z$ fiducial cross section and decay to a leptonic channel in $13 \mathrm{TeV} p-p$ collisions is measured to be $\sigma_{\text {fid }}=63.2 \pm 3.2$ (stat) \pm 2.6 (syst) \pm 1.5 (lumi) fb. When extrapolated to the total phase space, the total cross section is $\sigma_{\text {tot }}=50.6 \pm$ 2.6 (stat) \pm 2.0 (syst) \pm 0.9 (syst) \pm 1.2 (lumi) pb, in good agreement with the recent NNLO calculation [2] predicting $48.2_{-1.0}^{+1.1} \mathrm{pb}$.

\section{References}

1. LHC Higgs Cross Section Working Group Collaboration, J.R. Andersen et al. Handbook of LHC Higgs Cross Sections: 3. Higgs Properties, arXiv:1307.1347 [hep-ph]

2. M. Grazzini, S. Kallweit, D. Rathlev, M. Wiesemann, $W^{ \pm} Z$ production at hadron colliders in NNLO QCD, arXiv:1604.08576 [hep-ph] 